\title{
Highly nonlinear contact interaction and dynamic energy dissipation by forest of carbon nanotubes
}

\author{
Chiara Daraio, Vitali F. Nesterenko, ${ }^{a)}$ and Sungho Jin \\ Materials Science and Engineering Program, Mechanical and Aerospace Engineering Department, \\ University of California, San Diego, La Jolla, California 92093-0411
}

(Received 2 August 2004; accepted 6 October 2004)

\begin{abstract}
Mechanical response and energy dissipation of an array of carbon nanotubes under high-strain rate deformation was studied using a simple drop-ball test with the measurement of the dynamic force between the ball and forest of nanotubes. This convenient process allows extracting forcedisplacement curves and evaluating dissipated energy by the nanotubes. The contact force exhibits a strongly nonlinear dependence on displacement being fundamentally different than the Hertz law. The forest of vertically aligned nanotubes may be used as a strongly nonlinear spring in discrete systems for monitoring signal propagation speed, and as a microstructure for localized energy absorption. (C) 2004 American Institute of Physics. [DOI: 10.1063/1.1829778]
\end{abstract}

Strongly nonlinear elastic materials are of scientific and technical interest as their unique characteristics can be utilized as an energy-absorbing layered material for noise and shock wave mitigation and as nonlinear phononic crystals. ${ }^{1}$ The most famous example of such a nonlinearity is the Hertzian interaction law describing forces at one single contact between two linear elastic solids, where the forcedisplacement relationship has no linear part because of the variation of contact area with the force. ${ }^{2}$ Changing contact interaction is crucial for tailoring properties of strongly nonlinear phononic material, for example, for the tunability of the speed and shape of propagating signal. ${ }^{1}$ Another example of strongly nonlinear behavior can be found in some lowdensity materials, such as foams, cellular, or fibrous materials. ${ }^{3,4}$ The drawback of these materials is that when large deformations are involved, damage, crushing, or elastic buckling may dominate the behavior of these materials.

Since their discovery, ${ }^{5}$ carbon nanotubes (CNTs) have been explored for many potential technical applications. The mechanical response of individual nanotubes under axial and radial deformation has been studied extensively using theoretical and molecular-dynamics analysis. ${ }^{6-9}$ The elastic modulus $(E)$ has been theoretically calculated in agreement with experimental data to be very high, about $1 \mathrm{TPa} .^{10,11}$ Nonlinear elastic properties, stability, yielding, and fracture of CNTs were studied in Refs. 7-10 and 12-16 showing mechanical robustness of the nanotubes.

Behavior of bundles of nanotubes under pressure was studied in Refs. 17 and 18, and nonlinear penetration resistance was experimentally investigated for the forest of vertically aligned nanotubes. ${ }^{19}$ However, neither experimental data on the contact resistance of the forest of nanotubes under dynamic conditions nor their ability for energy storage/ dissipation at high-strain rate deformation are available.

In this letter, we present a simple method to determine the high-strain rate response and mechanical properties of a forest of vertically aligned CNTs. This allowed measurement and calculation of force-displacement relations for highstrain rate penetration process at the level of displacement as small as a few microns. The results of this work indicate that
CNTs, with their exceptional elastic stiffness combined with excellent resilience under large strain exhibit a strongly nonlinear behavior.

Arrays of carbon nanotubes were grown in a microwave plasma chemical vapor deposition (CVD) system consisting of a $2.45 \mathrm{GHz}$ microwave power supply and an inductively heated substrate stage. ${ }^{20,21}$ Prior to growth, $\sim 5 \mathrm{~nm}$ thick film of cobalt metal catalyst was evaporated onto a silicon (100) substrate. The samples were transferred to the growth chamber and heated to $765^{\circ} \mathrm{C}$ in hydrogen flowing at 200 standard cubic centimeters per minute ( $\mathrm{sccm}$ ) and held at 20 Torr. A $1 \mathrm{~kW}$ microwave plasma was subsequently ignited. Ammonia $\left(\mathrm{NH}_{3}\right)$ gas was then introduced to completely replace the hydrogen, and acetylene $\left(\mathrm{C}_{2} \mathrm{H}_{2}\right)$ was added 2 min later to start the nucleation and growth of nanotubes. The growth duration was $50 \mathrm{~s}$. The total gas flow rate and pressure was held constant at $200 \mathrm{sccm}$ and 20 Torr respectively. The $\mathrm{NH}_{3}: \mathrm{C}_{2} \mathrm{H}_{2}$ ratio was $3: 1$. The cobalt catalyst layer breaks up into islands on heating, thus inducing a growth of individual nanotubes. The local electric field in the microwave processing allows aligned growth.

The nanotubes had a narrow diameter distribution around $30 \mathrm{~nm}$ and were about $7 \mu \mathrm{m}$ in average length, a small portion of the nanotubes were much longer than the average. The estimated density is about $100 \mathrm{CNTs} / \mu \mathrm{m}^{2}$. A typical microstructure of CNTs is shown in Fig. 1(a). The nanotubes showed slight tangling near the top region, which could be due to their natural mechanical growth configuration or due to van der Waals force attraction of nearby nano-

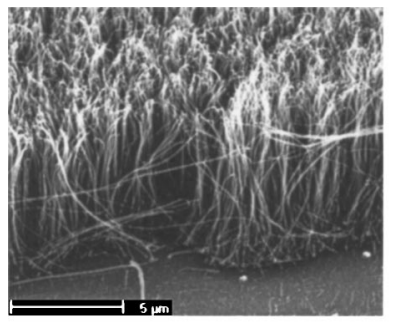

(a)

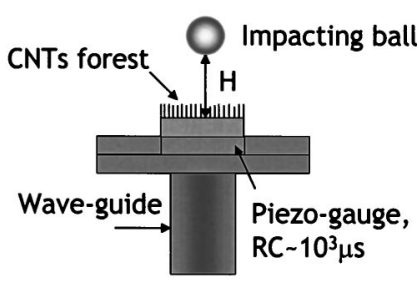

(b)
FIG. 1. (a) Forest of CNTs before impact and (b) experimental setup for measurement of contact force between the CNTs and the impacting ball. 


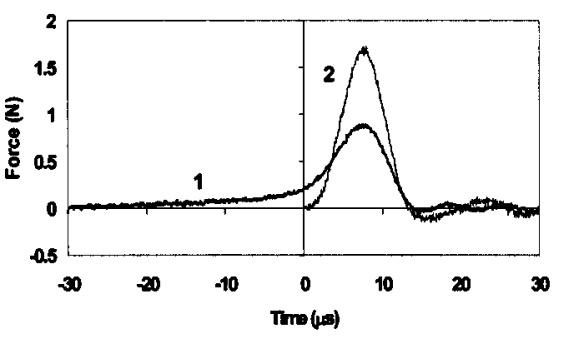

(a)

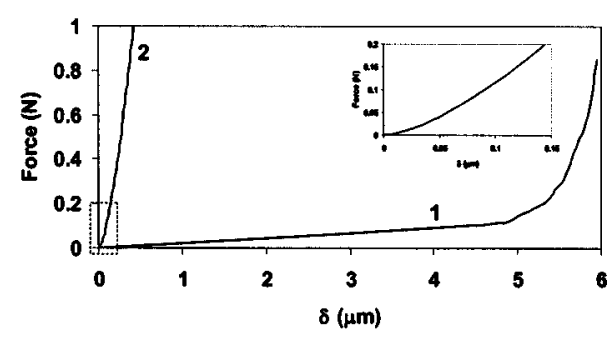

(b)

FIG. 2. (a) Experimental data for force vs time for contact interaction between the steel ball and the CNTs forest (curve 1) compared to that between the ball and bare Si wafer (curve 2). For convenience, comparison curves are centered at their corresponding maximum; (b) force-displacement curves for contact interaction of a ball with a forest of CNTs (curve 1) and Hertzian contact interaction of a ball with bare Si wafer [curve 2 based on Eq. (3)].

tubes. The presence of such entanglement may help to form a laterally connected nanotubes arrangement structure.

The experimental setup for measurement of mechanical response of the contact between the impacting ball and the array of nanotubes is presented in Fig. 1(b). It includes a calibrated piezosensor $\left(R C \sim 10^{3} \mu \mathrm{s}\right)$ connected to a Tektronix oscilloscope to detect force-time curves during dynamic interaction. Calibration was performed taking into account conservation of linear momentum. A piezogauge was placed on the top surface of a long, vertical steel rod (wave guide) imbedded at the bottom into a steel block to avoid possible waves reverberation in the system.

High-strain rate contact interaction was generated by an impact of a steel ball with $2 \mathrm{~mm}$ diameter dropped from a height of $2 \mathrm{~mm}$ at room temperature. The calculated velocity of impact was $0.20 \mathrm{~m} / \mathrm{s}$, the duration was about $37 \mu$ s ensuring the strain rate in the interval $10^{4}-10^{5} \mathrm{~s}^{-1}$. An essentially similar contact behavior was observed for impact velocities of 0.14 and $0.30 \mathrm{~m} / \mathrm{s}$. Scanning electron microscopy (SEM) analysis of deformed nanotubes was performed on a Phillips SEM operated at $30 \mathrm{kV}$.

The measured contact force between the steel ball and the forest of nanotubes versus time is shown in Fig. 2(a), curve 1. Repeated experiments demonstrate identical behavior of contact force. For comparison, the contact response on the bare Si wafer under identical impact conditions was also measured and presented in Fig. 2(a), curve 2. With the presence of nanotubes array, a dramatic change in the slope of contact force is evident in the first part of interaction process. A significant decrease of the maximal force caused by the deformation of the forest of nanotubes is also noticeable.

Contact force-displacement curve $(F-\delta)$ was constructed based on the measured dependence of force on time. Conservation of linear momentum allows an estimation of the ball velocity dependence on time, $u(t)$, using Eq. (1):

$$
m\left(u-u_{0}\right)=-\int_{t_{0}}^{t} F(t) d t .
$$

The velocity of impacting ball $u(t)$ can then be used for calculation of its displacement $\delta$ starting from $t_{0}$, which is the beginning of interaction between the ball and the forest of CNTs [-30 $\mu \mathrm{s}$ in Fig. 2(a), curve 1]

$$
\delta=\int_{t_{0}}^{t} u(t) d t .
$$

Dependence $(F-\delta)$ was obtained by comparison of experimentally detected force $F$ and calculated displacement $\delta$ at the same moment $t$. In calculations, the following values Downloaded 28 Sep 2006 to 131.215.225.9. Redistribution subject of parameters were used: mass $m=0.03 \mathrm{~g}$ and the initial impact speed $u_{0}=0.20 \mathrm{~m} / \mathrm{s}$. The interaction force between a ball and forest of nanotubes can be approximated by power laws with significantly different exponents for different ranges of displacements. In the interval $\delta \leqslant 4.8 \mu \mathrm{m}, F$ $=A \delta^{m}$ with $A=2.1 \times 10^{-2}$ and $m=1.07$, and in the interval $4.8 \mu \mathrm{m} \leqslant \delta \leqslant 6 \mu \mathrm{m}, \quad F=B \delta^{n}$ with $B=1.03 \times 10^{-7}$ and $n$ $=8.78$. Force $F$ is measured in Newtons and displacement $\delta$ in micrometers. The obtained data might also be used for estimating elastic properties of a densified forest of CNTs.

The resultant force-displacement curve is shown in Fig. 2(b), curve 1. For comparison, the Hertzian equation [Fig. 2(b), curve 2$]^{2}$ for ball-silicon wafer contact is also presented as described below

$$
F(\delta)=\frac{4 E_{1} E_{2} \sqrt{R} \delta^{3 / 2}}{3\left[E_{2}\left(1-\nu_{1}^{2}\right)+E_{1}\left(1-\nu_{2}^{2}\right)\right]},
$$

where $R$ is the radius of the ball, $\delta$ indicates the displacement during interaction, $\nu_{1}$ and $\nu_{2}$ are Poisson coefficients, and $E_{1}$ and $E_{2}$ are Young's moduli for $\mathrm{Si}$ and steel, respectively $\left(\nu_{1}=\nu_{2}=0.28, E_{1}=130 \mathrm{GPa}, E_{2}=207 \mathrm{GPa}\right)$.

It is clear from curves 1 and 2 in Fig. 2 that the nanotube layer exhibits a fundamentally different response of contact interactions as compared to the silicon surface. This nonHertzian behavior is revealed by initially weakly nonlinear force dependence on displacement in a relatively wide range of $\delta=0-5$ microns [compare insert in Fig. 2(b) for Hertz law for small $\delta$ and curve 1]. At larger displacements, the contact force [Fig. 2(b), curve 1] starts displaying a strongly nonlinear behavior in the remaining displacement range of 5-6 microns. The initial 5 micron displacement are accompanied by a relatively small increase in force, which might be related to an initial engagement of the ball surface with the taller tubes sticking out of the forest. As the ball continues to penetrate into the nanotubes, forest densification and deformation of the forest follow. The dramatic change in force slope observed in the last stage $\sim 1$ micron range of ball displacement prior to the peak deformation position. The velocity of the ball is zero at the peak of the $F-t$ curves in Fig. 2(a). The slope increase might be related to the continuously increasing volume density of nanotubes as the height of the compressed manotubes array is decreased further by the impacting ball (this contact behavior is qualitatively different from linear elastic materials which follow Hertz law). It is not clear if this highly nonlinear behavior is connected to the sideways interaction of the bending nanotubes. Another factor to consider is that as the upper part of nanotubes bends sideways, the remaining still-vertical portion of the same o AIP license or copyright, see http://apl.aip.org/apl/copyright.jsp 


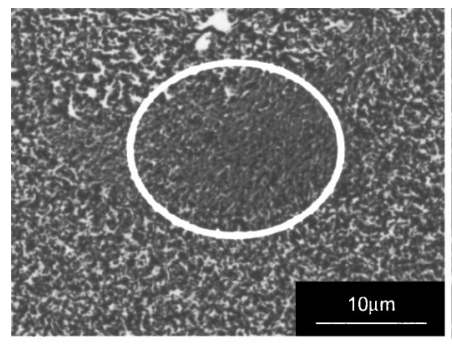

(a)

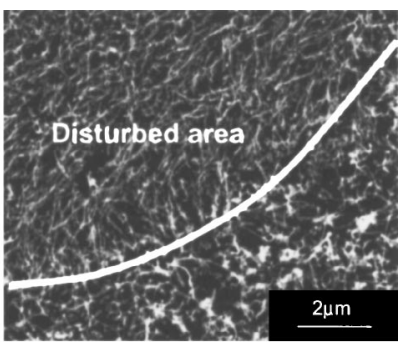

(b)

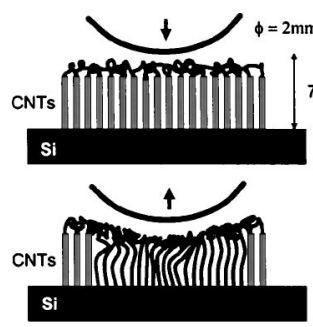

(c)

FIG. 3. (a) Heavily deformed impacted area shown by white circle; (b) interface between heavily deformed and undeformed region and (c) schematic diagram showing the initial and final stages in the nanotube array.

nanotubes gets shorter, thus requiring a much higher stress to deform. In order to clarify the mechanisms associated with the weakly nonlinear part of interaction, experiments with a very uniform height would be desirable. Strongly nonlinear materials, such as our nanotube forest, can be useful as nonlinear springs between inertial elements, and can be utilized to design "sonic vacuum"-type devices ${ }^{1}$ with possible applications for noise and shock wave mitigation and impact impulse transformation.

SEM analysis, [Fig. 3(a) and 3(b)] shows bent but not fractured nanotubes in the central heavily affected area of impact ( $\sim 20 \mu \mathrm{m}$ in diameter). It is not clear whether some of the observed bending is purely elastic or it contains permanent plastic deformation, for example, involving the formation of pentagon-heptagon-type defects. ${ }^{22}$ A schematic picture of interaction of the ball with forest of nanotubes is shown in Fig. 3(c). It is possible that elastic sideway displacements of the nanotubes (from the original vertically aligned position) are retained due to the after-impact entanglement or van der Waals force-type attractions between some of the adjacent locally contacting nanotubes. From the known average density of the nanotubes, the estimated number of nanotubes in the impacted area (20 $\mu \mathrm{m}$ diameter) is $\sim 3 \times 10^{4}$. The overall interacting volume associated with the densely populated nanotubes array of $7 \mu \mathrm{m}$ height in the deformed region is about $2.2 \times 10^{-15} \mathrm{~m}^{3}$.

The maximum force applied on this area is $0.9 \mathrm{~N}$. The estimation of ball displacement during active rebound stage based on the corresponding force dependence on time [Fig. 2(a), from maximum force to zero in curve 1] gives a value of about 0.4 microns [Fig. 3(c)].

The value of the coefficient of restitution at impact velocity of $0.20 \mathrm{~m} / \mathrm{s}(e=0.47)$ is calculated from the dependence of contact force on time [Fig. 2(a), curve 1] on the first (penetration) and second stages of interaction (rebound). This allows an estimate of the energy absorbed by the deformed carbon nanotube forest $E_{a}$ after impact as

$$
E a=\frac{m u_{0}^{2}}{2}\left(1-e^{2}\right) \text {. }
$$

Impact with a velocity of $0.20 \mathrm{~m} / \mathrm{s}$ results in absorbed energy equal to $0.47 \mu \mathrm{J}$ or an upper limit for volume density $235 \mathrm{MJ} / \mathrm{m}^{3}$ per initial volume of an impacted area of the forest of nanotubes. The corresponding localized energy on each nanotube gives a value of $0.015 \mathrm{~nJ}$, which is comparable to Ref. 18. This amount of energy can be related to the estimated maximum pressure on the contact ( $3 \mathrm{GPa}$ ) based on the maximal measured force $(0.9 \mathrm{~N})$ and diameter of the heavily deformed area (about $20 \mu \mathrm{m}$ ) [Fig. 3(a)]. This indicates that the forest of nanotubes can be utilized as a mechanical energy storage device at high-strain rate deformation as suggested earlier for reversible quasi-static compression at similar level of pressures. ${ }^{18}$

A different array of multiwall CNTs synthesized by dc Plasma $\mathrm{CVD}^{23}$ showed a dramatically different behavior.

In summary, it is shown that the dynamic deformation of a forest of CNTs exhibits a strongly nonlinear non-Hertziantype contact interaction law, which may be useful for the design of strongly nonlinear phononic discrete systems using the nanotube array as nonlinear springs. Such a nonlinear behavior allows tunability of properties of phononic devices due to the dramatic change of stiffness of contact interaction with applied static force. The results also reveal a significant level of energy absorption on high-strain rate deformation at high pressures on the order of a few GPa.

This work was supported by the NSF (Grant No. DCMS03013220). The authors wish to thank Dr. L. H. Chen and J. AuBuchon at UC San Diego for help.

${ }^{1}$ V. F. Nesterenko, Dynamics of Heterogeneous Materials (Springer, New York, 2001).

${ }^{2}$ K. L. Johnson, Contact Mechanics (Cambridge University Press, Cambridge, UK, 1985).

${ }^{3}$ L. J. Gibson and M. F. Ashby, in Cellular Solids (Pergamon, New York, 1988).

${ }^{4}$ M. Baudequin, G. Ryschenkow, and S. Roux, Eur. J. Biochem. 12, 157 (1999).

${ }^{5}$ S. Iijima, Nature (London) 354, 56 (1991).

${ }^{6}$ S. Iijima, C. Brabec, A. Maiti, and J. Bernholc, J. Chem. Phys. 104, 2089 (1995).

${ }^{7}$ B. I. Yakobson, C. J. Brabec, and J. Bernholc, Phys. Rev. Lett. 76, 2511 (1996).

${ }^{8}$ M. R. Falvo, G. J. Clary, R. M. Taylor, V. Chi, F. P. Brooks, S. Washburn, and R. Superfine, Nature (London) 389, 582 (1997).

${ }^{9}$ T. Belytschko, S. P. Xiao, G. C. Schatz, and R. S. Ruoff, Phys. Rev. B 65, 235430 1-8 (2002).

${ }^{10}$ C. Q. Ru, Phys. Rev. B 62, 9973 (2000).

${ }^{11}$ A. Pantano, D. M. Parks, and M. C. Boyce, J. Mech. Phys. Solids 52, 789 (2004).

${ }^{12}$ M. Arroyo and T. Belytschko, Mech. Mater. 35, 193 (2003).

${ }^{13}$ T. Ozaki, Y. Iwasa, and T. Mitani, Phys. Rev. Lett. 84, 1712 (2000).

${ }^{14}$ C. Wei, K. Cho, and D. Srivastava, Appl. Phys. Lett. 82, 2512 (2003).

${ }^{15}$ S.-P. Chan, W.-L. Yim, X. G. Gong, and Z. F. Liu, Phys. Rev. B 68, 075404 (2003).

${ }^{16}$ B. I. Yakobson, M. P. Campbell, C. J. Brabec, and J. Bernholc, Comput. Mater. Sci. 8, 341 (1997).

${ }^{17}$ M. J. Peters, L. E. McNeil, J. P. Lu, and D. Kahn, Phys. Rev. B 61, 5939 (2000).

${ }^{18}$ S. A. Chesnokov, V. A. Nalimova, A. G. Rinzler, R. E. Smalley, and J. E. Fisher, Phys. Rev. Lett. 82, 343 (1999).

${ }^{19}$ H. J. Qi, K. B. K. Teo, K. K. S. Lau, M. C. Boyce, W. I. Milne, J. Robertson, and K. K. Gleason, J. Mech. Phys. Solids 51, 2213 (2003).

${ }^{20}$ C. Bower, W. Zhu, S. Jin, and O. Zhou, Appl. Phys. Lett. 77, 830 (2000).

${ }^{21}$ C. Bower, O. Zhou, W. Zhu, D. J. Werder, and S. Jin, Appl. Phys. Lett. 77, 2767 (2000)

${ }^{22}$ B. I. Yakobson, Appl. Phys. Lett. 72, 918 (1998).

${ }^{23}$ C. Daraio, V. F. Nesterenko, J. AuBuchon, and S. Jin, Nano Lett. 4, 1915 (2004). 